\title{
Subcutaneous calcium heparin versus intravenous sodium heparin in treatment of established acute deep vein thrombosis of the legs: a multicentre prospective randomised trial
}

\author{
M G WALKER, J W SHAW, G J L THOMSON, J G R CUMMING, M LEA THOMAS
}

\begin{abstract}
One hundred patients with phlebographically proved acute deep vein thrombosis of the legs were prospectively randomised into two treatment groups to compare the safety and efficacy of subcutaneous calcium heparin versus intravenous sodium heparin admininstered by constant infusion pump. The dose of heparin was determined by daily measurement of the kaolin cephalin clotting time. Treatment was maintained for up to 14 days, after which phlebography was repeated. Of 49 patients who received subcutaneous calcium heparin, two showed an increase in thrombus size, while eight showed complete lysis. In the 47 patients who received intravenous sodium heparin thrombus increased in size in 13 while only one showed evidence of complete lysis. These differences were significant. There were no significant differences between the two groups in the incidence of serious complications, although almost half of those receiving intravenous heparin had some minor problem with the constant infusion pump and just over half of those receiving subcutaneous heparin had some bruising at the injection site.

This study showed that subcutaneous calcium heparin was more effective in helping lyse existing thrombus and preventing its propagation than intravenous sodium heparin.
\end{abstract}

\footnotetext{
Departments of Surgery and Radiology, Ninewells Hospital and Medical School, Dundee DD1 9SY

M G WALKER, CMH, FRCS, consultant vascular surgeon

JW SHAW, MB, FRCR, consultant radiologist

G J L THOMSON, MB, FRCS, research fellow in vascular surgery

J G R CUMMING, MS, FRCS, senior surgical registrar

Department of Radiology, St Thomas's Hospital, London SE1 7EH M LEA THOMAS, PHD, FRCR, senior physician in radiology

The trial was coordinated at Ninewells Hospital and Medical School and performed at Ninewells Hospital, King's College Hospital (Professor V V Kakkar, Mr G Murray), Derbyshire Royal Infirmary (Dr J Windebank), Royal Infirmary of Edinburgh (Mr C V Ruckley), Birmingham General Hospital (Mr N Dorricott), and Brighton General Hospital (Mr A Tanner).

Correspondence to: $\mathrm{Mr}$ Walker.
}

\section{Introduction}

Heparin, discovered in 1916 and first used as a therapeutic anticoagulant in 1936, is available as either sodium or calcium heparin. Differences between heparins have been observed, according to both the source and the salt, although not all of these are observed in vivo. ${ }^{12}$

Heparin may be administered intravenously, either by constant infusion or intermittent injection, or by intermittent subcutaneous injection. Of the intravenous routes continuous infusion is preferred because the incidence of haemorrhagic side effects is lower. ${ }^{34} \mathrm{~A}$ previous clinical trial showed that subcutaneous calcium heparin was more effective in treating established deep venous thrombosis of the calf veins than was sodium heparin given intravenously. ${ }^{5}$ This trial was designed to compare the efficacy and safety of these two methods of administration in the treatment of phlebographically established acute deep vein thrombosis at any site in the leg.

\section{Patients and methods}

One hundred patients with phlebographically proved acute deep vein thrombosis of the lower limbs were randomly allocated to receive either subcutaneous calcium heparin or intravenous sodium heparin by constant infusion pump. To be eligible for inclusion patients had to have thrombus with an unbroken extent of at least 5 centimetres evident in the calf, popliteal, femoral, or iliac veins. Patients with proved pulmonary embolism or in whom the thrombus was occlusive were excluded. Finally, the patient's condition was such that heparin treatment would ordinarily be advised. The predisposing factors in this group of patients are shown in table I, 65\% having undergone surgery.

Informed consent was obtained from all patients eligible for the trial. The patients' allocations were taken from sealed envelopes; the randomisation code was drafted using a standard random number table randomising in blocks of 10 .

\section{HEPARIN ADMINISTRATION}

Calcium heparin, from the same batch of hog intestinal mucosa, was kindly provided by Labaz:Sanofi UK Limited, each $0.8 \mathrm{ml}$ ampoule containing 20000 IU of heparin activity. Patients receiving calcium heparin 
were given $250 \mathrm{IU} / \mathrm{kg}$ subcutaneously, the dose being repeated every 12 hours and adjusted to maintain the kaolin cephalin clotting time at one and a half times to twice the normal value. Daily samples for this test were obtained five to seven hours after the morning dose. To avoid bruising as much as possible calcium heparin was injected using the technique of Griffith and Boggs. ${ }^{\circ}$

The intravenous heparin group received $250 \mathrm{IU} / \mathrm{kg}$ over 12 hours, the heparin being diluted in $50 \mathrm{ml}$ of normal saline. As in the other group, the rate of infusion was adjusted to maintain the kaolin cephalin clotting time at one and a half times to twice the normal value.

Treatment was maintained for up to $\mathbf{1 4}$ days, oral anticoagulation being introduced three days before the end of heparin therapy. Ascending phlebography was repeated on the final day of treatment, after which the subsequent dose of warfarin sodium was controlled using the prothrombin time, which was maintained at one and a half times to twice the normal value.

\section{ASSESSMENT}

Phlebography-Ascending phlebography (bilateral when necessary) was carried out using non-ionic contrast medium before entry into the trial to confirm the presence of a deep vein thrombosis and to assess the extent of thrombus formation. Phlebography was repeated on the final day of treatment. Thereafter the pretreatment and post-treatment paired phlebograms were assessed by a consultant radiologist (MLT), who had no knowledge of the patient, the treatment, or the centre from which the phlebograms had come.

Haematological-Haemoglobin concentration, packed cell volume, and platelet count were estimated on days 1, 7, and 14 during treatment. Kaolin cephalin clotting time was measured daily, five to seven hours after the morning dose.

Clinical-The patient's general condition was noted each day. In particular, the injection site was inspected twice daily, as were surgical wounds when present. Urine analysis was carried out on days 1, 7, and 14 . Discomfort at the site of injection was noted and any complications of treatment carefully recorded. Concurrent medication was noted.

Statistical analysis-The $\chi^{2}$ test was used to analyse the results. The difference in treatment response between the two groups was measured by deriving an index of effectiveness, obtained by subtracting the percentage showing an increase in the size of thrombus from the percentage undergoing complete lysis and reduction in size. The difference between the indices in the subcutaneous and intravenous heparin groups was calculated with a $95 \%$ confidence interval.

\section{Results}

Twenty five men, mean age 61 years (SD 11), and 25 women, mean age 63 years (16), were treated with subcutaneous calcium heparin, and $28 \mathrm{men}$, mean age 60 years (14), and 22 women, mean age 63 years $(15)$, were treated with intravenous sodium heparin. The incidence of the various predisposing factors did not vary significantly between the two groups (table I).

TABLE I-Predisposing factors to deep vein thrombosis

\begin{tabular}{lcc}
\hline & $\begin{array}{c}\text { Subcutaneous } \\
\text { calcium heparin }\end{array}$ & $\begin{array}{c}\text { Intravenous } \\
\text { sodium heparin }\end{array}$ \\
\hline Surgery & & \\
Gynaecological & 3 & 2 \\
Vascular & 2 & 0 \\
Abdominal & 17 & 4 \\
Orthopaedic & 7 & 5 \\
Other & 4 & 0 \\
Cardiac catheterisation & 1 & 1 \\
Tumours & 2 & 6 \\
Spontaneous & 7 & 11 \\
Medical inpatient & 7 & 50 \\
\hline Total & 50 & \\
\hline
\end{tabular}

Thrombus was unilateral in 39 of those receiving subcutaneous heparin and 40 of those receiving intravenous heparin. The distribution of thrombus was comparable between the two groups, 26 of the subcutaneous heparin group and 30 of the intravenous heparin group having major (above the knee) thrombi.

Three patients in the intravenous group had technically unsatisfactory post-treatment phlebograms and were therefore excluded from assessment. One patient in the subcutaneous group died during the study from septicaemia secondary to a subphrenic abscess; although he had completego his course of treatment, follow up phlebography was not possible. Nă patient was withdrawn from treatment during the study.

Efficacy-The comparison between the phlebograms taken before an $\mathbb{2}$ after treatment is shown in table II. More thrombi were lysed and fewer enlarged in the subcutaneous group than in the intravenous group. The difference in the index of effectiveness was $48 \%$ (95\% confidence interva $19.5 \%$ to $76 \cdot 5 \%$ ). Reduction in size or lysis of thrombus was not confined t $\$$ the calf veins (table III).

TABLE II-Independent phlebographic assessment comparing findings in phlebogram taken after treatment with those in pretreatment phlebogram

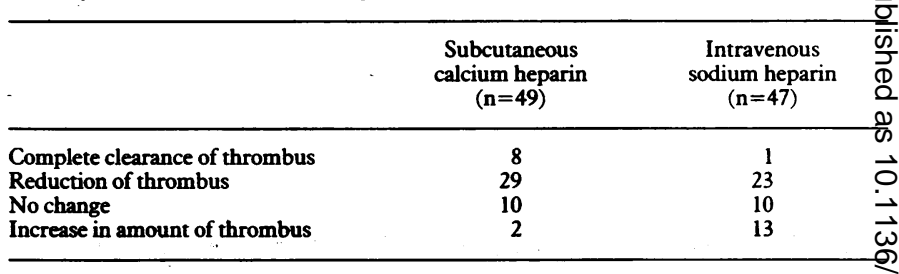

Overall $\chi^{2}=14 \cdot 17,2 \mathrm{df}, \mathrm{p}<0 \cdot 01 \cdot \chi^{2}$ for trend $=12 \cdot 47,1 \mathrm{df}, \mathrm{p}<0 \cdot 001$.

TABLE III-Distribution of thrombi showing lysis or reduction in size

\begin{tabular}{lcc}
\hline & $\begin{array}{c}\text { Subcutaneous } \\
\text { calcium heparin }\end{array}$ & $\begin{array}{c}\text { Intravenous } \\
\text { sodium heparin }\end{array}$ \\
\hline $\begin{array}{l}\text { Above knee (major) } \\
\text { Lysis }\end{array}$ & 2 & 0 \\
$\begin{array}{l}\text { Reduction } \\
\text { Below knee (minor) } \\
\text { Lysis } \\
\text { Reduction }\end{array}$ & 16 & 16 \\
\hline
\end{tabular}

Complications-Two patients in each group required a blood transfusio of two units because of a fall in haemoglobin concentration. Otherwise, ther were no major haemorrhagic complications, and the only significans difference between the two groups was bruising at the injection site in 27 of the subcutaneous group compared with only three of the intravenous group The bruises in the subcutaneous group ranged from 7 to $17 \mathrm{~mm}$ in diameter but in only two patients did they cause severe pain. The relatively higf incidence of bruising was due to a faulty injection technique which occurred: in the early stages of the trial with 15 patients. Minor complications at the injection site in the intravenous group were one case of infection, one of minor allergy, and two of thrombophlebitis. The incidence of minow pulmonary embolism did not differ significantly between the two groups occurring in two of the subcutaneous group and three of the intravenous group. Minor interruptions of treatment occurred in 21 patients in the intravenous group mostly because of mechanical problems with the infusion pump.

Dose of heparin-The mean daily and total requirements in those receivingu subcutaneous calcium heparin were both greater than in those receiving intravenous sodium heparin, although only the difference in mean daily dose was significant $(\mathrm{p}<0.02)$ (table IV).

\section{Discussion}

Subcutaneous injection of heparin has potential advantages ove intravenous injection. Intravenous administration greatly limits the $\mathrm{e}^{+}$ patients's mobility, and treatment may have to be interrupted if the patient needs to visit other hospital departments. Maintaining accurate dosage may also be a serious problem when mechanicap problems arise with the constant infusion pump. Subcutaneous administration avoids these difficulties.

Satisfactory anticoagulant activity was shown after low doso subcutaneous administration of both calcium and sodium salts by Thomas et $a l,{ }^{1}$ who also found lower plasma heparin concentration and a reduction of prolongation of the kaolin cephalin clotting time 
in those treated with calcium heparin. They therefore suggested that calcium heparin might be associated with fewer haemorrhagic complications. Their findings agreed with the earlier claim by Detrie et al that subcutaneous calcium heparin was associated with fewer local haemorrhagic side effects than the sodium salt. ${ }^{7}$ Recent comparative studies have, however, failed to confirm this finding and have not shown any difference between the two salts in efficacy or complications. ${ }^{28}$

Hirsh et al found that an adequate and stable anticoagulant effect could be obtained in most patients with two daily subcutaneous injections of calcium heparin, the therapeutic level being reached within two hours and the peak value at five hours. ${ }^{9}$ Clotting time remained within the therapeutic range for about 10 hours, and complications were minor.

Although many studies have shown the benefit and safety of small subcutaneous doses of heparin given as prophylaxis, few have used this method of administration to treat established acute deep vein thrombosis of the legs. ${ }^{510-12}$ The conventional method of treating acute deep vein thrombosis remains that of administering sodium heparin intravenously for several days and supplementing this with oral anticoagulation.

Our results, comparing the conventional intravenous route with the subcutaneous route in patients with deep vein thrombosis of the legs, showed a significant difference between the two groups in the number of thrombi undergoing complete lysis and in the number increasing in size during treatment, the differences favouring subcutaneous heparin. These results concur with those of Bentley et $a l$, who carried out a similar trial and found subcutaneous calcium heparin to be more effective in controlling extension of thrombi confined to the calf veins. ${ }^{5}$

The mechanism for the difference in the results between the two groups remains unclear, but Bentley et al suggested three possible explanations. Firstly, patients in the calcium heparin group may have received more heparin. Although this was not the case in their study, it was in ours (table IV), although the mean total doses were not significantly different. The greater dose was reflected in a higher

\begin{tabular}{|c|c|c|}
\hline & $\begin{array}{l}\text { Subcutaneous } \\
\text { calcium heparin }\end{array}$ & $\begin{array}{l}\text { Intravenous } \\
\text { sodium heparin }\end{array}$ \\
\hline $\begin{array}{l}\text { Daily dose } \\
\text { Total dose }\end{array}$ & $\begin{array}{c}29375(8354) \\
405435(134758)\end{array}$ & $\begin{array}{c}24384(12402)^{\star} \\
260617(13371)\end{array}$ \\
\hline
\end{tabular}

${ }^{\star} \mathrm{p}<0.02$.

mean daily kaolin cephalin clotting time during the course of the study (see figure). Secondly, the stimulus for thrombin generation may have differed between the two groups. As in the series studied by Bentley $e t a l$, a difference was unlikely as our groups were well matched for age, sex, and predisposing factors. The third possible mechanism relates to levels of circulating antithrombin III, a proteinase inhibitor whose presence is vital to the anticoagulant effect of heparin. Bentley et al measured antithrombin III values in 10 of their patients and found that the subcutaneous group had increased concentrations of antithrombin III, while those in the intravenous group had reduced concentrations. This finding matched that of a previous study in which the presence of heparin in the circulation for a prolonged period was associated with a reduction in antithrombin III concentrations, an effect not seen after a single injection. ${ }^{13}$ As the complex formed between heparin and antithrombin III is important in neutralising activated factor Xa and thrombin the lower levels of antithrombin III in the intravenous group may explain the results. We cannot confirm or refute this theory. A further possible explanation, as yet unsubstantiated, is that heparin given subcutaneously becomes defractionated, selectively allowing the low molecular effective fraction of heparin to be

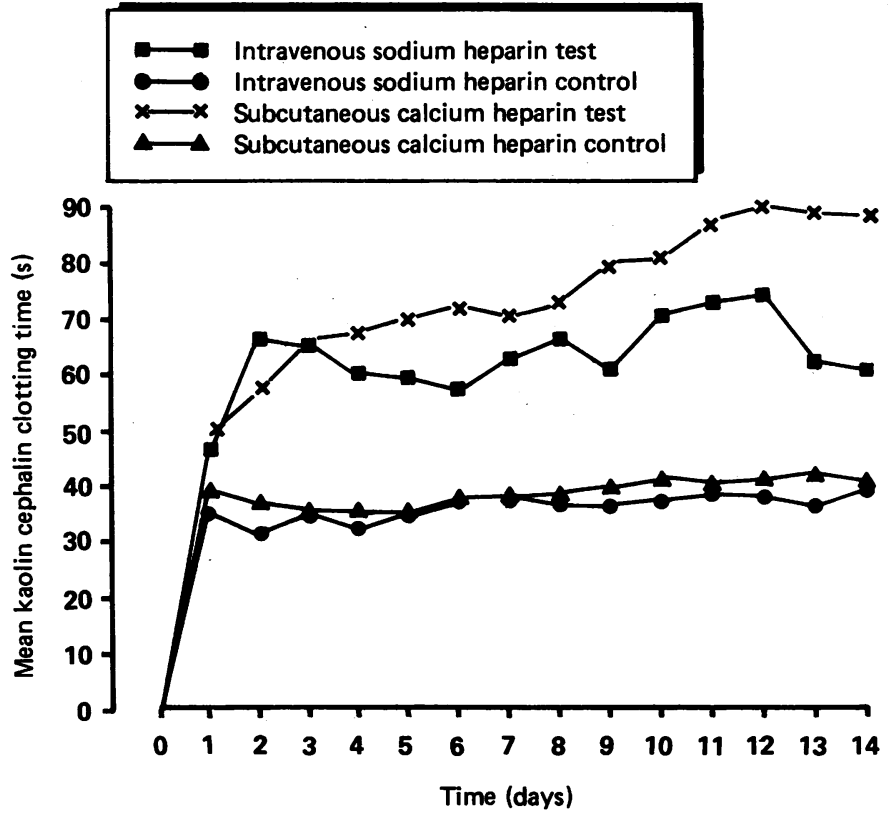

Comparison between sodium and calcium heparin mean daily kaolin cephalin clotting time values.

released. Recent work has also suggested that heparin administration may enhance fibrinolysis by potentiating urokinase type and tissue plasminogen activators. ${ }^{14} 15$

Complications in both our groups were similar, except for bruising at the injection site in those receiving subcutaneous calcium heparin. Most patients, however, were not upset by this and experienced minimal, if any, discomfort; only two complained of severe pain at the injection site. Minor interruptions of treatment occurred in 21 patients in the intravenous group but none of the calcium heparin group. The lack of interruption in the subcutaneous group may have contributed to the better results obtained, as might the greater mobility afforded to the subcutaneous group. The reason for the higher dosage requirement in the calcium heparin group remains unclear, but it was not associated with any significant increase in complications.

Our results are at variance with those of Hull $e t a l$, who recently reported a randomised trial of intravenous and subcutaneously administered heparin in the treatment of established proximal vein thrombosis, in which they found a higher incidence of recurrent thromboembolism in those treated subcutaneously. ${ }^{12}$ They failed to state, however, which salt was used in each group and whether the same batch of heparin was used throughout. Furthermore, all recurrences were in patients with an initial subtherapeutic anticoagulant response, suggesting a failure of adequate anticoagulant dosage in the subcutaneous group rather than a failure of the heparin per se. By contrast, we had no difficulty maintaining our patients within the therapeutic anticoagulant range, and patients in the subcutaneous group had, on average, higher daily kaolin cephalin clotting times than those in the intravenous group.

Our study has shown therefore that subcutaneous calcium heparin is more effective in helping lyse existing thrombus and preventing its propagation than is intravenous sodium heparin. This is true not only for minor thrombi below the knee but also for major thrombi above the knee. It is easier to administer, allows greater mobility during treatment, and is more acceptable to patients, nursing, and medical staff. On the evidence of this trial we feel that subcutaneous calcium heparin should be used as the first choice for managing established acute deep vein thrombosis of the legs, whether associated with pulmonary embolism or not.

This work was supported by Labaz:Sanofi UK Ltd and we gratefully acknowledge their assistance. We also thank $\mathrm{Mr} \mathrm{S}$ Ogston, lecturer in medical statistics, Ninewells Hospital, for his help in analysing the results. 


\section{References}

1 Thomas DP, Sagar S, Stamatakis JD, Maffei FHA, Erdi A, Kakkar VV. Plasma heparin levels after administration of calcium and sodium salts of heparin. Thromb Res 1976;9:241-8.

2 Lahnborg G, Bergstrom K. Clinical and haemostatic parameters related to thromboembolism and low dose heparin prophylaxis in major surgery. Acta Chir Scand 1975;141:590-5.

3 Salzman EW, Deykin D, Shapiro RM. The management of heparin therapy. Controlled prospective trial. N Engl I Med 1975;292:1046-50.

4 Glazier RL, Crowell EB. Randomised prospective trial of continuous vs intermittent heparin therapy. IAMA 1976;236:1365-7.

5 Bentley PG, Kakkar VV, Scully MF, et al. An objective study of alternative methods of heparin administration. Thromb Res 1980;18:177-87.

6 Griffith GC, Boggs RP. The clinical usage of heparin. Am $f$ Cardiol 1964;14:39-46.

7 Detrie P, Frieleux C, Dreux C, Vairel EG, Leger L. L'heparinate de calcium, ses proprietes et ses indications. Presse Medicale 1962;13:627.

8 Segesser von D, Gruber UF. Vergleich der Wirksamkeit von Natriumheparinat und Calciumheparinat zur Verhutung thromboembolischer Komplicationen. Aremeimittelforsch 1977, 27:2157-63.
9 Hirsch J, O'Sullivan EF, Gallus AS, Martin M. Evaluation of subcutaneous calcium heparin therapy in the treatment of thrombo-embolic disease. Med f Aust 1970;i:15-7.

10 Anderson G, Fagrell B, Holmgren $\mathrm{K}$, et al. Subcutaneous administration of heparin. randomised comparison with intravenous administration of heparin to patients with deep-veing thrombosis. Thromb Res 1982;27:631-9.

11 Krahenbuhl B, Simon CA, Bouvuer CA, et al. Traitement heparinique. Comparaison entre les voies d'administration intraveineuse et sous-cutanee. Schweiz Med Wochenschr 1979;109D 1322-5.

12 Hull RD, Raskob GE, Hirsch J, et al. Continuous intravenous heparin compared with intermittent subcutaneous heparin in the initial treatment of proximal-vein thrombosis. $N$ Eng? f Med 1986;315:1109-14

13 Marciniak E, Gockerman JP. Heparin induced decrease in circulating antithrombin III. Lancet 1977;ii:581-4.

14 Lijnen HR, Collen D. Stimulation by heparin of the plasmin-mediated conversion of single-chair to two-chain urokinase-type plasminogen activator. Thromb Res 1986;43:687-90.

15 Andra-Gordon P, Strickland S. Interaction of heparin with plasmino plasminogen: effects on the activation of plasminogen. Biochem 1986;25:4033-40.

(Accepted 9 March 1987)

\section{Randomised comparison of early versus late induction
in post-term pregnancy
KÅRE AUGENSEN, PER BERGSJ $\varnothing$, TORUNN EIKELAND, KJELL ASKVIK,
JOHANNES CARLSEN}

\begin{abstract}
In a prospective randomised study of mothers referred for prolonged pregnancy (around the 42nd week) 214 (group 1) were submitted to attempted induction of labour and 195 (group 2) assigned to continue for a further week without intervention. Strict selection criteria were used for the certainty of term. Mothers in group 2 were given regular non-stress tests to ensure fetal wellbeing, as were those in group 1 in whom induction failed. In group 1, $48(23 \%)$ out of 210 first attempted inductions failed. In group 2, $135(69 \%)$ of the births started spontaneously as compared with $38(18 \%)$ in group 1 . The mean duration of labour was 7.5 hours in each group. There was no significant difference in incidence of operative delivery, use of analgesics, or signs of perinatal asphyxia. Significantly more children in group 1 needed phototherapy for hyperbilirubinaemia. There was a clustering of births in the late afternoon and evening, which was most pronounced in group 1.

A policy of vigilant non-intervention up to the 44th completed week of pregnancy does not appear to jeopardise mother or fetus.
\end{abstract}

\section{Introduction}

Clinical decisions vary considerably when pregnancy continues beyond the accepted normal term. ${ }^{12}$ Systematic induction of labour has been justified as a means of averting placental insufficiency and fetal death. Another argument for induction is that good timing will ensure delivery during hours of optimal staffing and preparedness

\footnotetext{
Department of Obstetrics and Gynaecology, University of Bergen, Kvinneklinikken, Haukeland Sykehus, N.5021 Bergen, Norway

KÅRE AUGENSEN, MD, senior registrar (reservelege)

PER BERGSJ $\emptyset$, MD, PHD, professor of obstetrics and gynaecology

TORUNN EIKELAND, MD, junior registrar (assistentlege)

KJELL ASKVIK, MD, senior registrar (reservelege)

JOHANNES CARLSEN, MD, junior registrar (assistentlege)

Correspondence to: Professor Bergsiø.
}

for emergency treatment. As some $10 \%$ of pregnancies go beyond 420 completed weeks, even when strict criteria for reliability of 3 menstrual dates are employed, ${ }^{3}$ a rigorous policy of induction after term generates a large extra clinical workload.

In Norway the established practice for mothers who pass the 4200 week limit is to refer them to the maternity hospital for evaluation:From then on the course of action depends on local policy. In ouro hospital the induction policy was fairly liberal. We planned and conducted the following study in order to obtain a more rationalo basis for future decision making.

\section{Patients and methods}

Design of study - The ideal comparison would be induction at the end of 42 weeks (294 days) versus no intervention before spontaneous labour. This, however, was not feasible, as the risk of prolonged pregnancy is ultimately greater than that of any deliberate form of intervention. We decided on 303 days as the limit for non-intervention and designed a randomised study of immediate induction of labour in women referred for post-term pregnancy. versus induction one week later in those who had not delivered in the interim.

Criteria for inclusion - We included in the study only healthy women with normal pregnancies. Other criteria for inclusion were a single fetus in cephalic presentation; a duration of pregnancy of 290 to 297 days from the first day of the last menstrual period; and reliable dates. Reliable dates were defined as regular menstrual periods $(28 \pm 4$ day intervals) and clear=. recollection of dates. Use of contraceptive pills during the two months beforeN the last menstrual period was a cause for exclusion. Table I lists other reasons for exclusion.

Examination, randomisation, and management-Pregnant women who had not delivered by about 42 weeks were referred by their doctors. Aftefo scrutiny of the menstrual and pregnancy histories one of us performed a clinical examination, and if all criteria for enrolment were fulfilled ${ }^{+}$ randomisation (non-stratified) was done. The midwife consulted a list of random numbers, which was inaccessible to the participating physicians.으

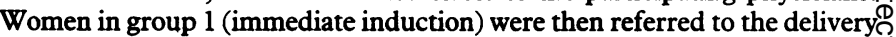
department for induction. Those assigned to group 2 (postponement of induction) were submitted to cardiotocographic non-stress tests on the day of referral (day zero) and again on day 3 or 4 if still undelivered. If birth had not occurred by day 7 labour was induced. In cases of failed induction in group 1 further management was as for group 2. For mothers who were stille undelivered after the attempted induction on day 7 management was left to clinical judgment. 